\title{
Um Ambiente para a Prática Remota de Aulas Laboratoriais de Física ( determinação da viscosidade de líquidos)
}

\author{
Cláudio Rodolfo Sousa de Oliveira \\ Universidade Estadual do Sudoeste da Bahia (UESB) \\ Estrada do Bem Querer, Km 04 \\ Vitória da Conquista - BA - Brasil \\ claudiorodolfo@yahoo.com.br \\ Adilson de Lima Pereira \\ Universidade Estadual do Sudoeste da Bahia (UESB) \\ Estrada do Bem Querer, Km 04 \\ Vitória da Conquista - BA - Brasil \\ adilson@uesb.br
}

\author{
Ivanor Nunes de Oliveira \\ Universidade Estadual do Sudoeste da Bahia (UESB) \\ Estrada do Bem Querer, Km 04 \\ Vitória da Conquista - BA - Brasil \\ ivanor@uesb.br \\ Hélio Lopes dos Santos \\ Universidade Estadual do Sudoeste da Bahia (UESB) \\ Estrada do Bem Querer, Km 04 \\ Vitória da Conquista - BA - Brasil \\ hls@uesb.br
}

Resumo Este artigo apresenta um ambiente que apóia a prática laboratorial de física à distância. Este ambiente é formado por uma maquete experimental, um circuito eletrônico que a automatiza, $e$ uma ferramenta que controla o circuito, possibilitando o uso dessa maquete remotamente. No ambiente a medição da viscosidade de líquidos é feita utilizando-se o método de Stokes, propiciando a coleta dos dados necessários ao cálculo da viscosidade do líquido em estudo, e a geração de um relatório, com todos os cálculos pertinentes à prática laboratorial. A ferramenta do ambiente é dividida em dois sistemas, que comunicam-se com uso da Application Programming Interface (API) Java chamada Remote Method Invocation (RMI): um sistema servidor desenvolvido em Java Standard Edition (JSE), que comanda o hardware do experimento , controlando o circuito eletrônico, e outro sistema Web desenvolvido em Java Server Pages (JSP), que é utilizado pelos alunos através da Internet, para manipulação e visualização do experimento em tempo real, ou pelos administradores do sistema para configurá-lo.

Palavras-Chave: Educação à distância, viscosidade de líquido, prática laboratorial.

\begin{abstract}
This paper presents an environment to support the remote laboratorial practice of physic. This environment is formed for an experimental model, a electronic circuit, and a software tool that controls the circuit, in order to enable the uses this model remotely, allowing to collects the necessary datas for the computes of the liquids' viscosity in study, and to generate a report with all important measurement from a laboratorial practice. The environment's tool is divided into two systems, that communicate each other using Application Programming Interface (API) Java called Remote Method Invocation: one system is developed with Java Standard Edition (JSE) and commands the hardware through an electronic circuit, and the another one is developed with Java Server Pages (JSF) and is used for the students over Internet to preview the experiment in real-time, or for system administrations to setup it.
\end{abstract}

Keywords: Distance learning, liquid's viscosity, laboratorial practice. 


\section{Introdução}

O experimento científico moderno caracteriza-se por uma grande quantidade de parâmetros mensuráveis, o que torna atual a utilização do computador para o controle do desenvolvimento do experimento, para o registro e elaboração dos resultados das medições e a apresentação dos dados obtidos numa forma cômoda para o pesquisador. Contudo, a automatização das medições e da elaboração dos dados do experimento hoje não se restringe apenas à pesquisa científica e, cada vez mais, tem sido introduzida também nas práticas laboratoriais dos cursos universitários, principalmente nos cursos de física.

Recentemente, na Universidade Estadual do Sudoeste da Bahia (UESB) foi implantado o Curso de Licenciatura em Física, modalidade de Ensino à Distância. Este curso tem como finalidade a capacitação de professores da rede pública do ensino médio. Para a modernização dos Laboratórios de Física Geral existentes na UESB foi criada uma linha de pesquisa ${ }^{1}$ cadastrada no Conselho Nacional de Desenvolvimento Científico e Tecnológico (CNPq) com o objetivo de automatizar os experimentos didáticos desenvolvidos nesta instituição pública de ensino superior.

A prática laboratorial de física na UESB tem sido, até agora, divida em duas etapas: 1) a coleta de dados durante as medições dos parâmetros físicos e 2) elaboração dos resultados dos correspondentes experimentos de Física Geral. O computador tem sido usado apenas na segunda etapa para os cálculos, geração de gráficos e visualização de resultados.

Tendo em conta que nos projetos técnicos [1] de criação das maquetes experimentais originais e nas modificações inovadoras realizadas estavam previstas as adaptações de sensores e de circuitos eletrônicos para a realização de medições de diversas grandezas físicas, a proposta de automatização dos experimentos existentes constitui um salto qualitativo na modernização dos laboratórios. Desta maneira, a concepção das maquetes experimentais dos Laboratórios de Física Geral está em correspondência com a proposta de automatização das medições e da elaboração dos resultados dos experimentos de Física geral e nelas podem ser acoplados os sistemas de automatização e controle.

Não há dúvida de que a utilização de sensores e circuitos eletrônicos contribuiria para aumentar o grau de precisão das medições dos parâmetros físicos, entretanto este uso não resolveria o seguinte problema.

A prática laboratorial de física continuaria divida em

${ }^{1}$ http://dgp.cnpq.br/buscaoperacional/detalhegrupo.jsp?grupo=7490105 2CJT4EL. duas etapas. Desta maneira, o computador continuaria a ser utilizado apenas na segunda etapa, para os cálculos, geração de gráficos e visualização de resultados.

Para resolver o problema apresentado, propomos a utilização do computador em todo o âmbito do experimento laboratorial, desde a coleta de informações até a análise de resultados. Neste caso, em primeiro lugar, o processo será realizado de forma integrada, mais segura e dinâmica no tocante a alguns erros que podem acontecer, principalmente durante a coleta de dados. Em segundo lugar, a automatização das medições e da elaboração dos dados do experimento de física, proporcionará mais tempo aos estudantes para: a complementação das aulas teóricas, o aprofundamento dos conhecimentos de métodos e técnicas do experimento, o estudo de aparelhos de pesquisa científica e familiarização com os mesmos, dominar diferentes métodos de pesquisa experimentais, dominar métodos matemáticos e gráficos dos resultados obtidos e também a avaliação das medições realizadas.

Desta forma, o objetivo principal deste trabalho é criar um ambiente que realize os cálculos para a determinação da viscosidade de um líquido pelo método de Stokes, utilizando uma ferramenta de software e uma maquete experimental automatizada do Laboratório de Física Molecular e Termodinâmica da UESB.

Dentre os objetivos deste ambiente destacam-se:

- Desenvolver uma maquete experimental para o calculo da viscosidade de líquidos, utilizando o método de Stokes.

- Automatizar esta maquete com auxílio de um circuito eletrônico, para a manipulação da mesma através do computador, necessitando da mínima intervenção humana na realização dos experimentos.

- Criar uma ferramenta de software composta por 2 módulos: 1) Um que manipule a parte eletrônica da maquete experimental automatizada, que persista os dados necessários a recuperação das informações do sistema em um banco de dados e que forneça todas as operações necessárias a uma aplicação cliente; e 2) outro que permita aos alunos do Curso de Física realizarem o Trabalho de Laboratório No. 4 intitulado "Determinação da viscosidade de um líquido com o auxílio de uma maquete experimental automatizada" [2], gerar o relatório deste experimento em consonância com o modelo proposto no anexo do referido trabalho de laboratório,. e permitir aos professores alterarem facilmente as informações necessárias ao funcionamento do ambiente, tudo isso remotamente.

$\mathrm{O}$ artigo está estruturado da seguinte forma: a seção 2 apresenta os fundamentos teóricos sobre a viscosidade dos líquidos, o método de Stokes para a determinação da 
viscosidade; a seção 3 aborda os trabalhos relacionados como os ambientes para educação a distancia: Modular Object Oriented Dynamic Learning Environment (MOODLE), TelEduc, e-Proinfo e AulaNet; a seção 4 descreve os dispositivos que constituem a maquete experimental, aborda as funcionalidades do sistema, como são feitos à persistência dos dados, como é a arquitetura lógica de comunicação entre os sistemas e como é realizada a transmissão de vídeo; a seção 5 descreve a implementação das funcionalidades do sistema que controla o circuito eletrônico e do sistema e; por fim, há as considerações finais e indicativos para trabalhos futuros.

\section{Coeficiente de viscosidade de líqui- dos}

A viscosidade [3] é uma força volumétrica de atrito interno que aparece no deslizamento de camadas fluidas umas sobre outras, dando origem a tensões tangenciais. Como resultado deste deslizamento, surge uma força dirigida em sentido oposto ao da velocidade do movimento.

São conhecidos muitos métodos de determinação da viscosidade. Neste experimento utiliza-se o método de Stokes, baseado na medição da velocidade de queda livre de esferas de pequenas dimensões num líquido viscoso.

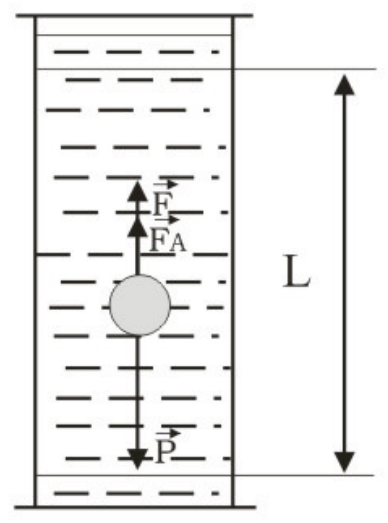

Figura 1: Forças atuantes nas esferas que caem num líquido[2].

Sobre uma esfera que cai livremente num meio viscoso atuam, na direção vertical, três forças (Figura 1): a força de gravidade $\vec{P}=\rho_{1} \cdot V \cdot \vec{g}$, a força de empuxo hidrostático $\vec{F}_{A}=\rho_{2} \cdot V \cdot \vec{g}$ e a força de resistência da viscosidade $\vec{F}=6 \pi \cdot \eta \cdot R \cdot \vec{v}$, onde $V=\frac{4}{3} \pi R^{3}$ é o volume da esfera, $\rho_{1}$ e $\rho_{2}$, são as densidades do materi- al da esfera e do líquido, $\eta$ é o coeficiente de viscosidade do líquido, respectivamente.

De acordo com a segunda lei da dinâmica, temos:

$$
m \frac{d v}{d t}=\left(\rho_{1}-\rho_{2}\right) \cdot V \cdot g-6 \pi \cdot \eta \cdot R \cdot v
$$

ou, tendo em conta o volume da esfera e a sua massa $m=\rho_{1} V$, obtemos:

$$
\frac{d v}{d t}=\frac{\left(\rho_{1}-\rho_{2}\right) \cdot g}{\rho_{1}}-\frac{9 \eta \cdot v}{2 R^{2} \rho_{1}}
$$

A força de atrito (ou de resistência de viscosidade) depende da velocidade e cresce com o aumento desta. Conseqüentemente, a aceleração da esfera irá diminuir e, finalmente, esta adquire tal velocidade pela qual a aceleração torna-se igual a zero. A esfera irá cair no líquido com movimento uniforme. No instante inicial $v=0$, depois a velocidade começa a aumentar, a aceleração diminuirá com o tempo e o incremento da velocidade diminuirá, mas esta seguirá aumentando constantemente. Tendo em conta a equação (2), é evidente que a velocidade não pode ser maior que o valor

$$
v_{0}=\frac{\left(\rho_{1}-\rho_{2}\right) \cdot 2 R^{2} g}{9 \eta}
$$

Se $v=v_{0}$, temos que $\frac{d v}{d t}=0$, pois a velocidade não pode seguir aumentando.

Da equação (3), temos que

$$
\eta=\frac{\left(\rho_{1}-\rho_{2}\right) 2 R^{2} g}{9 v_{o}}
$$

Desta maneira, a determinação da viscosidade $\eta$ consiste da medição da velocidade $v$ e do raio da esfera $R$.

A equação (4) é justa somente quando a esfera cai num meio sem fronteiras. Se a esfera cai ao longo do eixo de um tubo de raio $R_{0}$, então é necessário ter em conta a influência das paredes transversais do tubo. A fórmula para a determinação do coeficiente de viscosidade, tendo em conta esta influência, tem o seguinte aspecto:

$$
\eta=\frac{D^{2} g}{18 L}\left(\frac{\rho_{1}-\rho_{2}}{1+2,4 \frac{R}{R_{0}}}\right) t,
$$

onde D é o diâmetro da esfera.

A automatização do experimento, onde as maquetes 
experimentais estão ligadas ao computador, teve um grande salto qualitativo e quantitativo com o surgimento da rede da Internet. Os canais de ligação entre os computadores ficaram acessíveis, confiáveis e baratos. Surgiram os chamados laboratórios à distância, cujos usuários poderiam realizar as suas pesquisas encontrando-se a distâncias significativas. Estas possibilidades foram utilizadas, não somente na organização das pesquisas científicas, mas também dos laboratórios didáticos remotos $[4,5,6$, 7]. Tudo isto veio contribuir para o surgimento da educação à distância nas áreas científicas e técnicas.

\section{Trabalhos correlatos}

O resumo analítico apresentado abaixo sobre alguns ambientes virtuais de aprendizagem serviu para definir a ferramenta desenvolvida para o auxílio a educação a distância $(\mathrm{EaD})[8]$.

Foram analisados importantes ambientes de aprendizado como o MOODLE, AulaNet, TelEduc e e-Proinfo. Nenhum destes tem o mesmo escopo de trabalho do ambiente desenvolvido que é voltado para a prática laboratorial no ensino da física, mas de modo geral todos possuem a mesma finalidade, prover um ambiente virtual de aprendizado para auxilio da educação à distância. As principais características destes ambientes podem ser vistas a seguir.

MOODLE [9] é sistema desenvolvido de forma colaborativa que permite a criação e a administração de cursos na Web. Este sistema começou a ser idealizado no início da década de 90 por Martin Dougiamas, que conhecia muitas pessoas em escolas e instituições, as quais gostariam de fazer melhor uso da Internet, mas não sabiam como iniciar, devido à grande quantidade de ferramentas tecnológicas e pedagógicas existentes na época. Martin gostaria de proporcionar a essas pessoas uma alternativa gratuita e livre, que pudesse introduzi-las ao universo on-line.

Por ser um sistema de código aberto, livre e gratuito, os usuários podem baixá-lo, usá-lo, modificá-lo e distribuí-lo seguindo apenas os termos estabelecidos pela $G e$ neral Public License (GPL). O sistema possui ferramentas de comunicação, avaliação, disponibilidade, administração e organização de conteúdos.

AulaNet [10] foi criado pela Universidade Católica do Rio de Janeiro (PUC-Rio), é utilizado na criação e manutenção de cursos apoiados em tecnologia da Internet e pode ser utilizado tanto para ensino a distância como para complementação às atividades de educação presencial e treinamento de profissionais. Várias disciplinas de graduação e de pós-graduação da PUC-Rio o utilizam como complemento às suas atividades, assim como professores e alunos de universidades de todo o país. Este sistema também vem sendo utilizado por empresas interessadas em modernizar, agilizar e baratear o treinamento de seus funcionários.

O sistema implementa a capacidade de personalização da interface e da estrutura navegacional, além disso, disponibiliza nos cursos os seguintes serviços: comunicação (e.g., grupo de interesse, grupo de discussão, contato com o professor e debate), administrativos (e.g., agenda, notícias do curso, cadastro de instrutores, matrícula e mensagens automáticas), avaliação (e.g., teste, resultado do teste, projeto, resultado do projeto, exercício e resultado de exercício), didáticos (e.g., o de aula, livro texto, demonstrações, bibliografia) e gerais (e.g., tutorial sobre Internet, home-page de alunos e busca).

TelEduc [11] é um ambiente para a criação, participação e administração de cursos na Web. Esse ambiente foi concebido tendo como alvo o processo de formação de professores para informática educativa. Este sistema foi desenvolvido de forma participativa, ou seja, todas as suas ferramentas foram idealizadas, projetadas e depuradas segundo necessidades relatadas por seus usuários. As suas principais características são: a facilidade de uso por pessoas não especialistas em computação, a flexibilidade quanto ao modo de usá-lo e um conjunto enxuto de funcionalidades.

O sistema possui um amplo conjunto de ferramentas de comunicação como o correio eletrônico, grupos de discussão, mural, portfólio, diário de bordo, bate-papo, etc.

Assim como o MOODLE, este sistema também é um software livre podendo ser redistribuído e modificado sob os termos da GPL.

E-Proinfo [12] foi desenvolvido pela Secretaria de $\mathrm{EaD}$ do Ministério da Educação (MEC). O ambiente foi desenvolvido para complementar o programa educacional ProInfo que visava introduzir tecnologias de informação e comunicação, nas escolas públicas do Brasil, como ferramenta de apoio ao processo de ensino-aprendizagem. $\mathrm{O}$ ambiente do MEC não necessita de infra-estrutura para sua instalação, pois o sistema fica instalado no servidor do próprio MEC. As instituições públicas cadastradas podem acessar o ambiente usando qualquer computador. O e-ProInfo já foi usado para a formação de 50 mil alunos, a maioria funcionários públicos, em 235 cursos de aperfeiçoamento profissional.

Este ambiente virtual permite a concepção, administração e desenvolvimento de ações de apoio ao processo de ensino-aprendizagem. Para isso contem os recursos: fórum, videoconferência, bate-papo, e-mail, quadro de avisos, notícias e biblioteca. Há também um conjunto de recursos disponíveis para apoio às atividades dos participantes, como tira-dúvidas, avisos, agenda e diário. 


\section{Ambiente de EaD para a Prática Laboratorial de Física}

O desenvolvimento deste trabalho teve como objetivo atender as novas necessidades de realização da prática laboratorial de física. Neste sentido, era necessário o desenvolvimento de um ambiente de EaD que controlasse os mecanismos necessários à coleta dos dados e que ao mesmo tempo fornecesse uma interface via Internet para esta manipulação e visualização do experimento.

O ambiente de controle do experimento de viscosidade é formado pela integração de vários sistemas que se comunicam. Numa análise geral há três estruturas (camadas) bem distintas: 1) a maquete experimental, 2) o dispositivo eletrônico que controla a maquete experimental e 3) o sistema de software que controla o dispositivo eletrônico.

\subsection{Descrição do Hardware do Experimento}

O hardware do experimento é composto por uma maquete experimental, e pelos mecanismo que possibilitam o controle desta maquete através de um computador como: motor de passo, e receptores e transmissores de luz infrared, para automatizar a coleta dos dados, e um circuito eletrônico conectado no computador por uma porta paralela, para o manuseio da maquete.

\subsubsection{Maquete Experimental Automatizada}

A maquete experimental automatizada apresentada na Figura 2, foi projetada e construída pelo Prof. Dr. Ivanor Nunes de Oliveira. A maquete é constituída das seguintes partes: 1) Base metálica com pés niveladores; 2) Tubo de vidro transparente instalado verticalmente, que contém o líquido em estudo; 3) Sistema de retirada das esferas depositadas no fundo do tubo; 4) Tubo suporte de descarga das esferas acumuladas; 5) Haste metálica vertical com plataforma horizontal; 6) Sistema distribuidor individual de esferas; 7) Sistema de regulagem de altura da coluna de líquido; 8) Conjunto de sensores de passagem das esferas dentro da coluna líquida (Diodo emissor de luz/Foto-transistor) separados por uma distância $L$; 9) Hardware para controle (drive) do motor de passo; 10) Motor de passo.

A base metálica com pés niveladores contém um furo central onde está fixado um suporte tubular de conexão com o sistema de retirada das esferas que caem na superfície do líquido em estudo e são depositadas na extremidade do tubo de vidro transparente ligada a este sistema.

O sistema de retirada das esferas é formado por um compartimento cilíndrico ligado a dois registros de esfera. Mantendo-se, inicialmente, o registro superior aberto, as esferas depositadas no compartimento podem ser retiradas fechando-se este registro e abrindo-se o registro inferior. Tal procedimento permite a retirada das esferas, mantendo-se o nível original da coluna líquida. A mudança do líquido em estudo pode ser feita, de forma prática, soltando-se o sistema de retirada das esferas do seu encaixe para a lavagem do tubo. A haste metálica vertical parafusada na base pode ser ajustada radialmente.

Desta maneira, a plataforma horizontal fixada na haste é posicionada de forma que as esferas caiam no centro do tubo que contém o líquido em estudo, com o auxílio do sistema distribuidor de esferas. Os sensores de infravermelho instalados e separados por uma distância conhecida captam o momento em que a esfera interrompe o raio infravermelho (InfraRed - IR) gerando um pulso de +5 Volts, que é enviado à porta paralela do computador, possibilitando a captura do evento por uma aplicação que manipula esta porta.

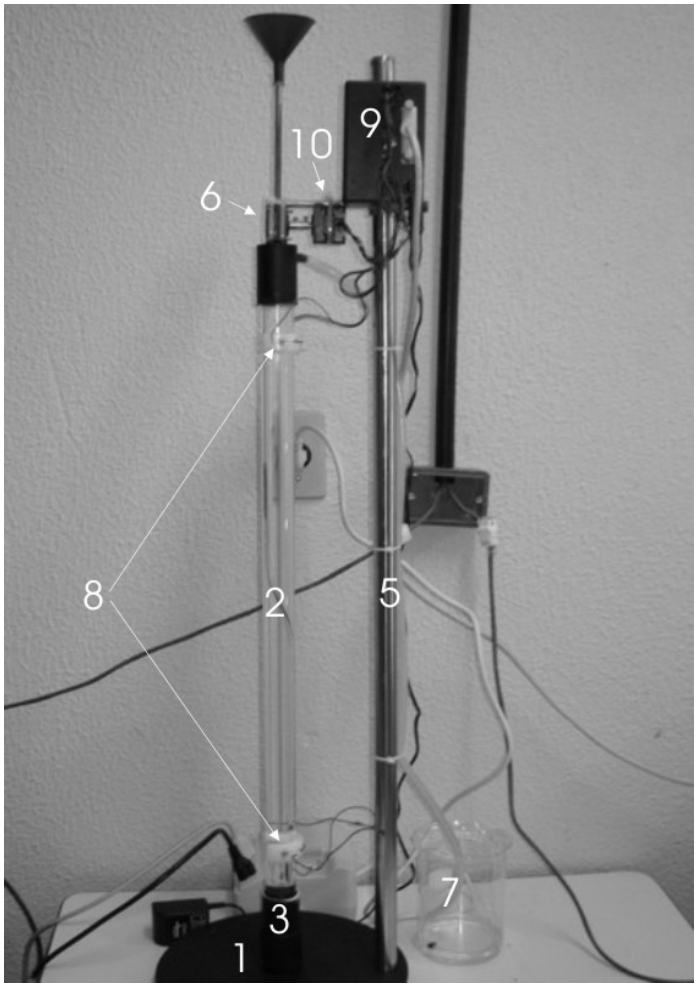

Figura 2: Fotografia da maquete experimental automatizada.

O hardware de controle e medição da maquete experimental é composto por um drive de motor de passo e por dois sensores de passagem (um superior e outro inferior) formados por um par de diodos emissores de luz (Light Emission Diode - LED) IR e um par de fototransistores receptores.

\subsubsection{Porta Paralela}


A porta paralela [13], também chamada de (Line Print Terminal - LPT), é uma interface de comunicação entre o computador e outros periféricos como, por exemplo, uma impressora. A comunicação com a LPT é realizada com o auxílio do conector de 25 pinos (conector padrão DB25), ligado diretamente à placa-mãe. Na Figura 3 ver-se o sentido de transmissão de dados de cada pino do conector.

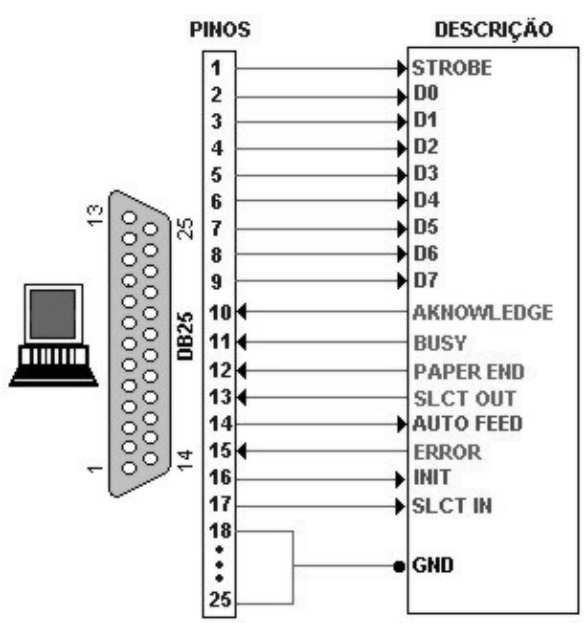

Figura 3: Significado de cada pino do conector DB25.

Os pinos 1, 14, 16 e 17 da porta paralela são próprios para o controle de impressoras, os pinos 2 a 9 enviam dados brutos para um dispositivo ligado a porta, os pinos $10,11,12,13,15$ são utilizados para que o computador receba dados de um dispositivo externo e os pinos 18 a 25 são utilizados para aterramento da porta.

A porta paralela é utilizada como meio que interliga a maquete experimental ao computador. Sinais específicos são usados para enviar comandos que fazem girar o motor de passo, enquanto outros sinais captam dados do dispositivo quando as esferas passam pelos sensores de detecção de passagem.

\begin{tabular}{c|c|c|lcc}
\hline Pinagem & Nome & Direção & \multicolumn{2}{|l}{ Função neste projeto } \\
\hline 2 à 5 & $\begin{array}{c}\text { D1 à } \\
\text { D4 }\end{array}$ & Saída & $\begin{array}{l}\text { Controle do motor de } \\
\text { passo }\end{array}$ \\
\hline 7 & D6 & Saída & $\begin{array}{l}\text { Alimentação do LED IR } \\
\text { inferior }\end{array}$ \\
\hline 10 & D9 & Entrada & $\begin{array}{l}\text { Alimentação do LED IR } \\
\text { superior }\end{array}$ & $\begin{array}{l}\text { Status foto- } \\
\text { transistor inferior }\end{array}$ \\
\hline 11 & D10 & Entrada & $\begin{array}{l}\text { Status foto- } \\
\text { transistor superior }\end{array}$ \\
\hline 18 a 25 & Ground & - & Aterramento & \\
\hline
\end{tabular}

Tabela 1: Sinais de controle do projeto pela porta paralela.
O motor de passo é controlado pelos pinos 2 a 5, e a passagem pelos sensores infravermelho são detectadas pelos pinos 10 e 11 como pode ser visto na Tabela 1 .

O Java é portável porque seu compilador não gera um código de máquina, e sim um código intermediário, que sempre é interpretado para a plataforma utilizada no momento da execução. Com isso, perde-se o acesso direto ao hardware do computador, que só seria possível com a geração deste código de máquina especifico para a arquitetura utilizada. Para que o Java consiga comunicar-se com o hardware da porta paralela é necessário o uso de sua API chamada Java Native Interface (JNI) [14]. Ela permite a Java Virtual Machine (JVM) interoperar de forma bidirecional com aplicações e bibliotecas desenvolvidas em outras linguagens de programação.

$\mathrm{Na}$ ferramenta utilizaram-se as rotinas do projeto Parport $^{1}$ desenvolvidas na linguagem de programação $\mathrm{C}++$ e embutidas no arquivo parport.dll. Para burlar esta deficiência do Java, Parport fornece uma classe chamada ParallelPort, onde a aplicação Java invoca os métodos desta classe para leitura ou escrita na porta e esta classe executa o método correspondente na aplicação em $\mathrm{C}++$, ficando esta operação transparente para o programador.

\subsubsection{Motor de Passo}

Os Motores de passos [15] são dispositivos mecânicos eletromagnéticos que podem ser controlados digitalmente com uso de um hardware específico ou com uso de softwares. Na Figura 4 tem-se um exemplo de como é construção física de um controle para motor de passo.

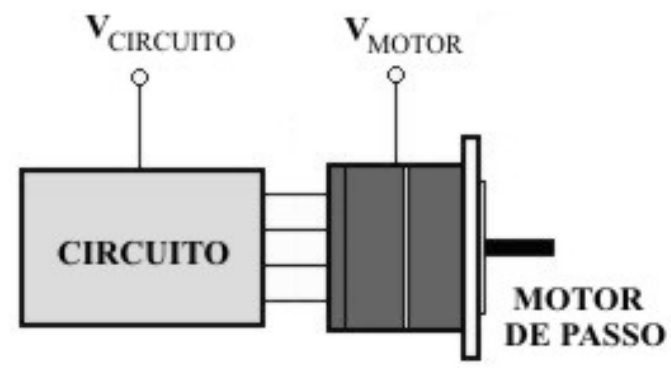

Figura 4: Bloco lógico e eletrônico de um motor de passo.

Circuito é a parte do circuito eletrônico que comanda o motor de passo via comandos enviados pela ferramenta. Sua fonte de alimentação ( $\left.\mathrm{V}_{\text {CIRCUITO }}\right)$ assim como a fonte do motor de passo $\left(\mathrm{V}_{\text {МотоR }}\right)$ é de 12 Volts. Esta separação é apenas lógica, no circuito eletrônico do projeto existe apenas uma fonte de alimentação.

A lógica empregada para que o motor gire corretamente e no tempo certo é implementada via software. $\mathrm{O}$ circuito apenas envia os dados do software para o motor

\footnotetext{
${ }^{1}$ http://www.geocities.com/Juanga69/parport.
} 


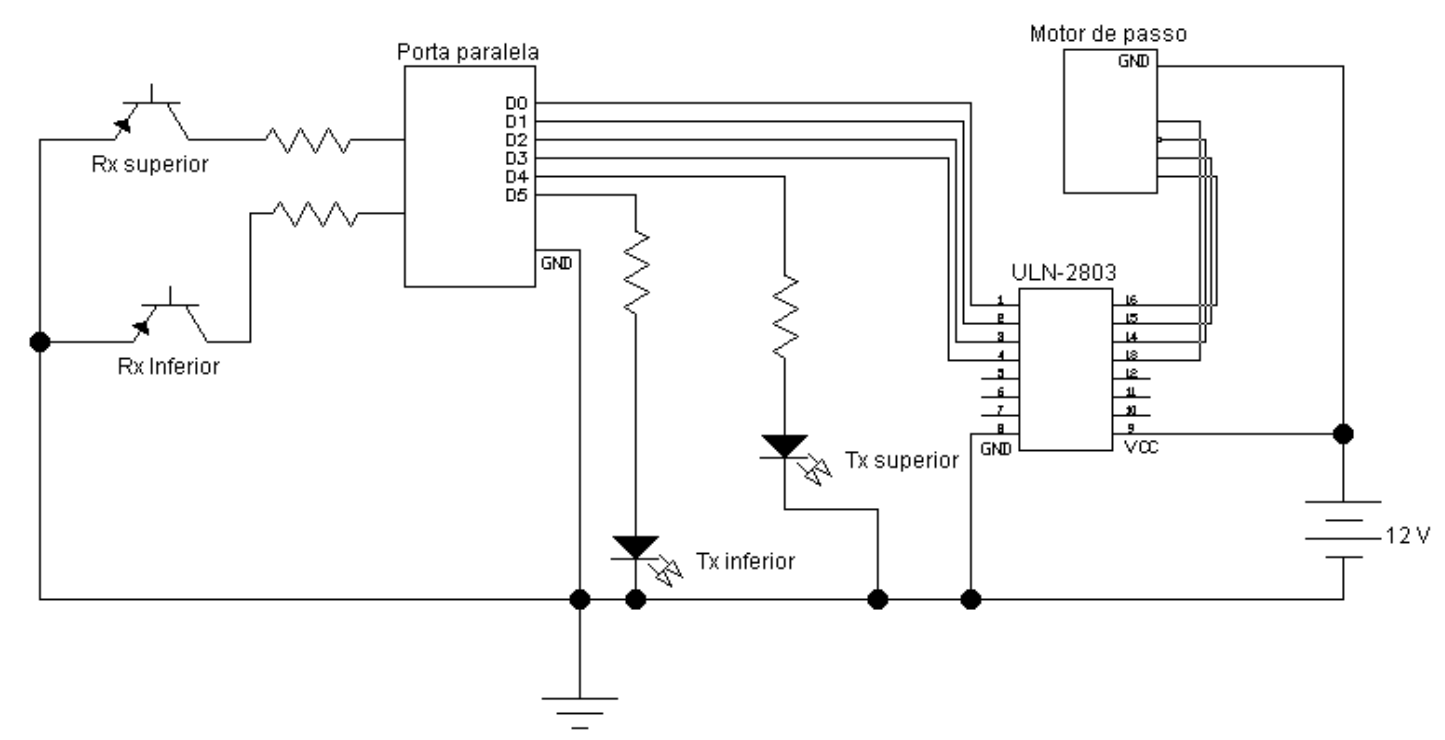

Figura 5 : Circuito eletrônico da maquete experimental.

não realizando nenhum processamento. Utiliza-se o CI ULN-2803 para isolar o computador do motor evitando assim uma possível sobre-tensão, que pode vir a danificar a entrada LPT do computador.

Existem motores de passo com várias escalas de ângulos diferentes. Os motores menos precisos giram tipicamente $90^{\circ}$ por passo, enquanto os motores de maior precisão são capazes de girar entre 1.8 e $0.72^{\circ}$ por passo. Neste experimento, o motor de passo é utilizado para mover o distribuidor de esferas, que as solta no líquido em estudo, como é previsto pelo método de Stokes. Esta tarefa é realizada via internet pelos usuários à distância pela aplicação $\mathrm{Web}^{1}$. O motor de passo utilizado no experimento tem uma precisão mediana girando $7,5^{\circ}$ por passo. Sendo assim, são necessários 48 passos para que ele dê uma volta de completa.

\subsubsection{Receptores e Transmissores De IR}

O sensor de detecção de passagem da esfera é formado por um LED IR $^{2}$ (transmissor) [15] e um fototransistor sensível à luz $\mathrm{IR}^{3}$ (receptor). O LED quando alimentado, transmite luz IR, que não é visível a olho nu, em todas as direções. O Foto-transistor quando iluminado por uma luz IR conduz corrente elétrica entre sua ligação Emissor-Coletor (EC) funcionando assim como uma chave acionada via luz IR.

\footnotetext{
${ }^{1}$ Software que é executado a partir de um navegador de internet.

${ }^{2}$ O LED IR utilizado neste experimento tem a especificação LD 271.

3 O Transistor utilizado neste experimento tem a especificação BP103B3.
}

No experimento existem dois sensores destes que ficam presos ao tubo da maquete experimental a uma distância previamente estabelecida, um superior e outro inferior. Com a distância conhecida e detectando-se o tempo que a esfera gasta para perfazer o caminho entre eles, é simples obter a velocidade de queda, haja vista que o movimento da esfera é uniforme no líquido.

Quando a esfera atravessa o feixe de luz emitido pelo LED, o Foto-transistor pára de receber a luz que o faz conduzir corrente, interrompendo a condução. Esta ação gera um pulso no pino onde está ligado o sensor, de duração igual ao tempo necessário para a esfera cruzar o feixe de luz. Com isso uma aplicação que leia a porta paralela neste instante pode detectá-lo.

Ao interromper o sensor superior é disparado um $t i$ mer que é parado quando o sensor interrompe o sensor inferior. Se a esfera se desviar e não cruzar o sensor superior ou cruzar apenas o sensor superior e no período definido na entrada TIMEOUT da sessão [PARAMETROS] do arquivo de configuração (ver Seção 4.4.2) não cruzar o inferior, este resultado é descartado, partindo-se para os demais.

\subsubsection{Circuito Eletrônico}

O circuito eletrônico que comanda a maquete experimental pode ser visto na Figura 5. Este circuito foi elaborado pelo Prof. Msc. Adilson de Lima Pereira e é constituído por um motor de passo que solta as esferas no líquido em estudo, um CI ULN-2803 para dar o ganho de corrente necessário ao funcionamento do motor e evitar sua ligação direta com a porta paralela do computador, 
uma fonte de alimentação de 12 Volts para alimentar o circuito e o motor de passo, um par de LEDs IR e dois foto-transistores sensíveis a esta luz [15].

Os pinos onde estão ligados os foto-transistores ficam constantemente conduzindo corrente porque estão recebendo a luz do LED. Ao passar uma esfera e interromper o feixe de luz IR, o Foto-transistor deixa de conduzir, com isso um pulso de 0 Volts é empregado na porta paralela.

Resistores de 100 Ohms são utilizados nos dispositivos ligados diretamente ao computador para limitar a corrente que passa por este equipamento, a fim de não os danificá-lo.

\subsection{Implementação da Ferramenta}

A ferramenta é formada por dois sistemas: 1) um Servidor criado em Java Standard Edition (JSE) [16] e 2) outro Cliente criado em JSP. O sistema Cliente é dividido em dois módulos, o módulo Usuário que é o utilizado pelos estudantes do curso de física, cadastrados para a manipulação do aparelho e o módulo Administrador, que é utilizado para a administração geral do sistema, utiliza- do normalmente por um professor ou funcionário do Laboratório de Física.

Ao entrar no site qualquer usuário, incluindo os não autenticados, tem acesso a algumas informações, como obter o guia de laboratório [2] para este experimento, contendo fundamentos teóricos sobre a viscosidade, perguntas de controle relativas ao tema e a bibliografia recomendada. Também estão disponíveis algumas informações, como o horário de acesso ao experimento, não sendo permitida a sua realização fora deste horário.

Existe um formulário de autenticação para fazer o chaveamento entre o módulo Usuário e Administrador do sistema Cliente. Após a autenticação, de acordo com o tipo de usuário autenticado, este é direcionado ao módulo Usuário ou Administrador, sendo possível o acesso ao módulo Usuário a partir do módulo Administrador, mas não o contrário.

\subsubsection{Funcionalidades da Ferramenta}

As funcionalidades do sistema Cliente da ferramanta podem ser vistas na Figura 6, pelo diagrama de Caso de Uso da Linguagem de Modelagem Unificada (Unified Modeling Language - UML) [16].

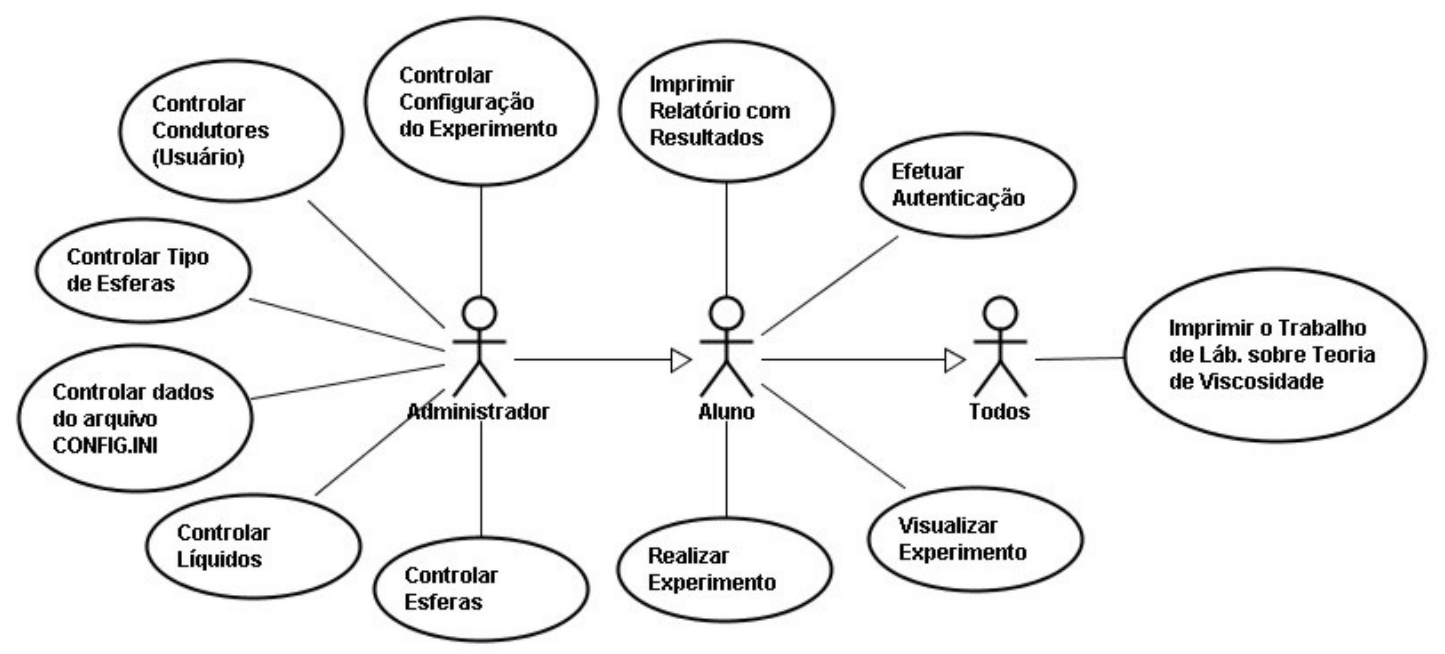

Figura 6 : Diagrama de Caso de Uso da UML do Sistema Cliente.

$\mathrm{O}$ ator ${ }^{1}$ intitulado como $\underline{\text { Todos }}$ representa qualquer pessoa que acesse o site do projeto, sendo a ela permitido apenas visualizar e imprimir guia de laboratório para este experimento.

$\mathrm{O}$ ator Aluno representa todos os alunos do curso de física que podem realizar o experimento via internet, para isso ele deve estar cadastrado na base de dados do siste-

\footnotetext{
${ }^{1}$ Tem o papel de estimular, solicitar ou receber ações e eventos do sistema.
}

ma e efetuar a autenticação para garantir a sua identidade. Uma vez autenticado, o aluno pode obter os resultados mediante o uso do experimento. Esta atividade é chamada de prática de laboratório. Depois da realização desta prática, pode-se gerar um relatório com todos os dados e cálculos provenientes das coletas realizadas e imprimi-lo para ser entregue ao professor da disciplina. Como estes dados são armazenados em banco de dados, não é necessário a imediata impressão do material, ficando o mesmo 
disponível a qualquer momento para consulta ou impressão, mediante prévia autenticação.

$\mathrm{O}$ ator Administrador representa aquelas pessoas responsáveis pela manutenção geral do sistema. Normalmente este tipo de usuário é representado por funcionários e professores do curso de física. Para este usuário também é necessário a autenticação prévia no sistema, para utilizálo. As tarefas básicas inerentes a este usuário são os controles dos registros de: esfera, tipo de esfera, líquido, configuração do experimento, condutor (usuários do sistema) e parâmetros do arquivo CONFIG.INI.

\subsubsection{Camada de Persistência dos Dados}

O banco de dados utilizado no ambiente foi do tipo Relacional [17] que é o tipo de banco de dados mais utilizado atualmente, porque sua linguagem de consulta de dados (Structured Query Language - SQL) é muito simples e intuitiva. Para a persistência dos dados optou-se pelo Sistema de Gerenciamento de Banco de Dados (SGBD) conhecido como Firebird $1.5^{1}$, porque seus requisitos mínimos de funcionamento não exigem um hardware sofisticado, além de ser rápido e gratuito.

Há informações que servem como parâmetros para a aplicação, e seu uso torna mais flexível a configuração do sistema. Para estas informações optou-se pela armazenagem em um arquivo de configuração do tipo $\operatorname{Ini}^{2}$ chamado CONFIG.INI. Não se optou pelo armazenamento em banco de dados porque os dados não são excluídos ou inseridos, apenas alterados esporadicamente. Na Figura 7 pode se ver o conteúdo do arquivo de configuração CONFIG.INI que está localizado no computador servidor do Laboratório de Física.

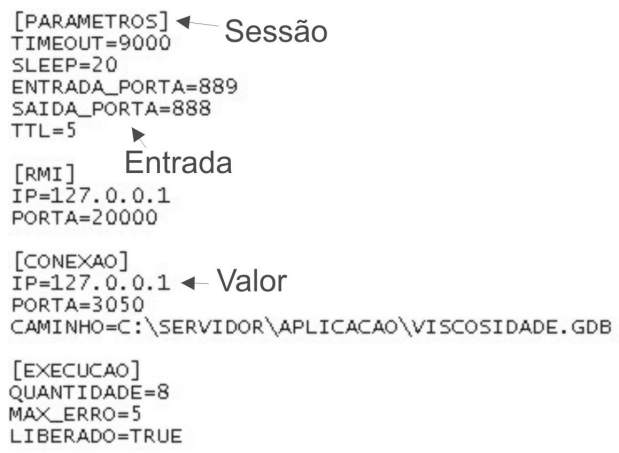

Figura 7: Arquivo de configuração CONFIG.INI.

Um arquivo do tipo Ini é constituído de três partes importantes. A primeira parte é constituída pelas sessões, elas ficam entre colchetes e não podem se repetir num mesmo arquivo. As sessões utilizadas no arquivo $\mathrm{CON}$ -

${ }^{1}$ http://www.firebird.org.

${ }^{2}$ Arquivo de inicialização do Sistema Operacional Microsoft Windows.
FIG.INI são [PARAMETROS], [RMI], [CONEXAO] e [EXECUCAO]. A segunda é formada pelas entradas, na mesma sessão não devem existir duas entradas com mesmo nome. A terceira parte é constituída pelos valores que é localizado no arquivo pela tupla única (sessão, entra$d a$ ). Não existe regra para a formação dos valores, sendo a semântica dada por uma aplicação que o capture.

\subsubsection{Arquitetura Lógica de Comunicação}

Um diagrama da arquitetura lógica de comunicação entre os diversos sistemas envolvidos no processo pode ser visto na Figura 8. Ela é lógica porque as ligações entre os sistemas não acontecem necessariamente de forma direta. Para os sistemas, a informação trafega desta maneira, mas na realidade existem diversos dispositivos de hardware entre eles.

No Laboratório de Física Geral da UESB encontra-se o computador utilizado como servidor do projeto de automatização dos laboratórios. O experimento automatizado, o motor de passo e os sensores são ligados a este computador pela porta paralela. Neste servidor encontra-se também o SGBD e o arquivo do banco de dados chamado "VISCOSIDADE.GDB". A comunicação entre o banco de dados e a aplicação se dá pela porta 3050, que é a porta padrão de comunicação com o SGBD Firebird (configurável).

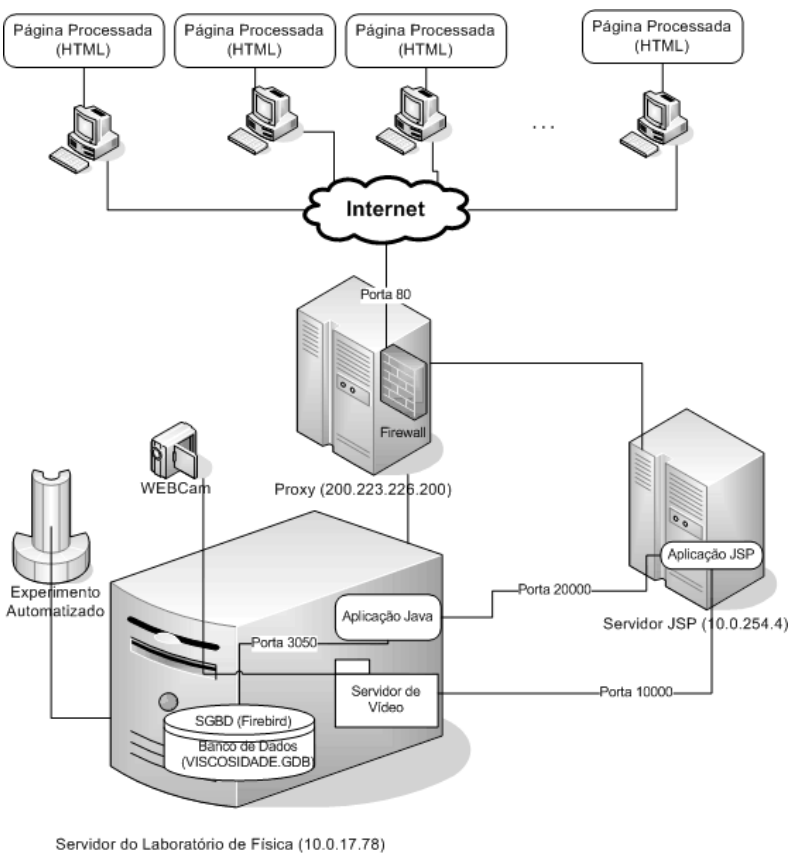

Figura 8: Diagrama de arquitetura lógica de comunicação.

Neste computador servidor do Laboratório de Física funciona o servidor de vídeo que é responsável por prover a imagem da Câmera Web (webcam) para os usuários 
pela internet pela porta 10000 (configurável). Neste servidor também funciona o sistema Servidor que capta os dados do experimento.

Há um servidor $W e b^{1}$ na Unidade Organizacional de Informática da UESB (UINFOR) que serve para processar as páginas Java Server Pages (JSP) [18] dos sistemas $w e b$ da Universidade. Neste servidor encontra-se o sistema Cliente (módulos Usuário e Administrador) da ferramenta. Este sistema Cliente comunica-se com o sistema Servidor localizado no computador servidor do Laboratório de Física pela porta 20000 (configurável).

Todos os parâmetros sinalizados anteriormente como configuráveis podem ser alterados no arquivo de configuração CONFIG.INI ou da janela "ArquivoIni" do módulo Administrador do sistema Cliente.

\subsubsection{Transmissão de Vídeo}

A transmissão de vídeo é feita com o software servidor de Vídeo conhecido como "Codificador do Windows Media 9 Series". Para que ele funcione corretamente no computador servidor, o mesmo deve ter instalado o Windows Media Player (WMP) ${ }^{2}$, porque este codificador é um plugin $^{3}$ para o WMP. Os usuários que pretendem visualizar o vídeo, devem ter um player compatível com o protocolo Multimedia Messaging Service (MMS) [19], no qual o vídeo é transmitido pela rede.

Este servidor possui inúmeras opções, sendo a utilizada neste projeto a opção "Transmissão de um evento ao vivo". Para que isto seja possível, é necessário que a webcam esteja corretamente instalada no computador servidor, no qual está instalado o servidor de Vídeo.

$\mathrm{Na}$ configuração pode-se optar por transmitir vídeo e/ou som. A webcam é detectada automaticamente, não necessitando de nenhum ajuste extra. Deve-se informar também a porta pela qual o servidor disponibilizará o serviço. Para este projeto foi escolhida a porta 10000 . Depois disto, deve-se informar a taxa de transmissão de vídeo. A nossa escolha foi a taxa de $128 \mathrm{Kbps}^{4}$, que fornece 15 quadros por segundo de 320x240 pixels. Pode-se optar em salvar o vídeo em disco, mas para este projeto não é interessante, pois o ambiente é previsível e imutável.

O servidor de Vídeo informa quais são os usuários que utilizam o serviço no momento e seus respectivos endereços do Internet Protocol (IP). Com este servidor

\footnotetext{
${ }^{1}$ Servidor que responde páginas HyperText Markup Language (HTML) ao navegador requisitante.

${ }^{2}$ http://www.microsoft.com/windows/windowsmedia

${ }^{3}$ Extensão que adiciona novas funcionalidades a um software

${ }^{4}$ Unidade de medida de transmissão de dados sendo $1 \mathrm{Kbps}=1$ Kilobit por segundo.
}

também é possível controlar, pelo endereço IP, quem pode ou não utilizar o serviço.

\subsubsection{Sistema Servidor}

O sistema Servidor, cuja interface pode ser visualizada na Figura 9, é executado ininterruptamente no computador servidor do Laboratório de Física. Neste sistema encontra-se praticamente toda a implementação dos métodos que o sistema Cliente irá utilizar. Este sistema é responsável pela manutenção dos dados no banco de dados, criação e destruição dos objetos instanciados no sistema Servidor pelo sistema Cliente, controle do experimento automatizado (leitura dos sensores e movimentação do motor de passo), assim como prover todas as funcionalidades necessárias ao sistema Cliente.

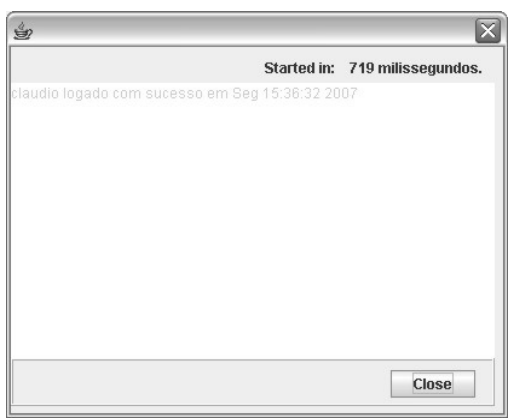

Figura 9: Tela do sistema Servidor.

As principais classes deste sistema são Transacao, FactoryManager, Resultado e Lib.

A classe Transacao implementa as funcionalidades referentes a persistência e busca dos dados de todas as janelas do sistema Cliente. Os métodos desta classe realizam as seguintes operações: gravar os dados no BD provenientes da inclusão ou alteração de um registro, excluir dados, verificar se a tabela manipulada está vazia, buscar o valor de um campo específico da tabela, contar o número de registros de uma tabela, gerar o código a ser utilizada na inclusão de um novo registro e realizar um lockup de um campo que consiste em buscar um valor de um campo de outra tabela baseado em sua chave localizada na tabela de origem. Como a classe é genérica, é necessário informar na construção do objeto qual tabela ele irá manipular.

A classe FactoryManager é responsável por gerenciar os objetos instanciados pelo sistema Cliente. Ela mantém uma tabela $h a s h^{5}$ com a referência dos objetos criados por ela. Quando ela recebe uma requisição de um objeto, verifica se o mesmo já existe através do código único

\footnotetext{
${ }^{5}$ É uma estrutura que permite associar uma chave a um valor e, posteriormente, ter acesso ao valor a partir de sua chave associada.
} 


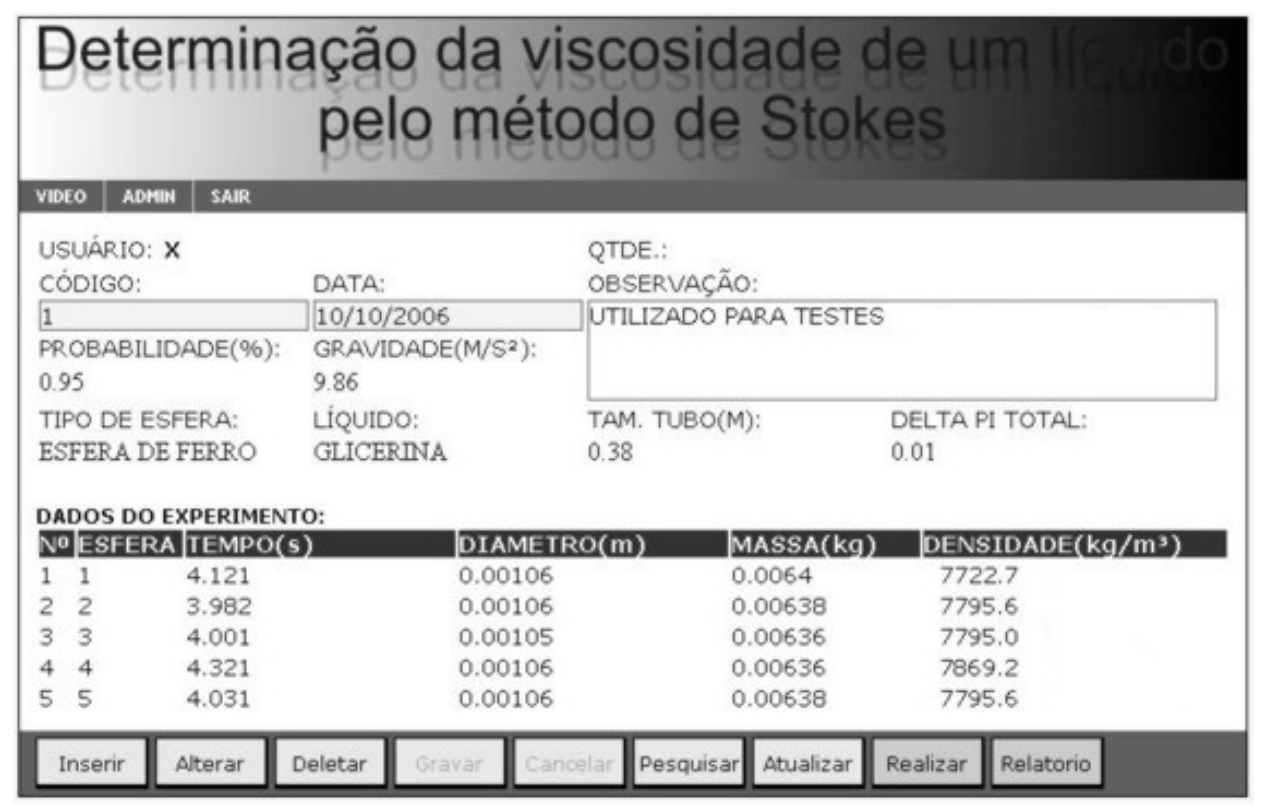

Figura 10 : Janela que controla os dados dos experimentos.

utilizado para identificá-lo na tabela, se ele não existir, um novo objeto é criado, referenciado na tabela e disponibilizado para o sistema Cliente, se ele existir apenas o último passo é executado. Quando o usuário sai do sistema Cliente ele invoca nesta classe do sistema Servidor os métodos de destruição dos objetos desreferenciando-os da tabela e excluídos os objetos da memória do computador.

A classe Resultado é a responsável pela captura dos dados e comando do motor de passo da maquete experimental. O método de coleta de resultados da classe possui um laço que envia pulsos para girar o motor de passo. A cada pulso é verificado se a esfera interrompeu o sensor superior, quando isto acontece o laço é interrompido parando assim o motor, um timer é iniciado aguardando que a esfera cruze o segundo sensor, quando isso ocorre o dado é gravado no BD e parte-se para a próxima coleta realizando toda a operação novamente.

A classe Lib realiza operações diversas. Entre estas operações destacam se verificar se o horário é apropriado para o usuário utilizar o experimento e aguardar um tempo pré-determinado antes de executar a próxima instrução (sleep) que é utilizada para aplicar os passos ao motor.

As únicas informações que este sistema apresenta na tela são o horário em que foi iniciado, o tempo necessário para iniciar o serviço e os usuários autenticados.

\subsubsection{Sistema Cliente}

O sistema Cliente foi criado utilizando JavaScript ${ }^{1}$,

\footnotetext{
${ }^{1}$ Linguagem de Script semelhante na linguagem de programação Java.
}

Cascade Sheet Style (CSS), HTML e principalmente JSP. Este sistema implementa as telas que os usuários podem acessar de qualquer navegador com acesso à internet. Este sistema também faz todo o controle de validação de dados a serem enviados para ao sistema servidor. A comunicação entre os sistemas Cliente e Servidor é realizada com uso da Interface de Aplicação (Application Programming Interface - API) do JSE conhecida como Remote Method Invocation (RMI) [20]. Esta API permite a invocação de métodos remotamente de uma aplicação Java por outra aplicação Java. Com isso, o sistema Cliente invoca os métodos de objetos instanciados do sistema Servidor onde estão implementados os métodos.

\subsubsection{Módulo Usuário}

Este módulo é utilizado pelos usuários do tipo user (normalmente estudantes do curso de física) para a manipulação e visualização do experimento, impressão de relatórios referentes à prática laboratorial.

A janela Experimento da Figura 10 manipula os experimentos dos usuários, sendo que cada usuário pode visualizar apenas os seus próprios experimentos.

Se o aluno estiver em um horário permitido para uso do experimento e sua cota de utilização (valor normalmente 1 referente à quantidade de experiências que cada

estudante pode realizar) o botão Realizar estará habilitado para a coleta dos dados. Uma vez coletados os dados, o botão Relatório estará habilitado para que o relatório com o resultado de todos os cálculos possa ser visto e impresso. 
Há também neste formulário a opção vídeo, que permite o usuário ver o experimento funcionando em tempo real pela webcam instalada no computador servidor do Laboratório de Física. Na Figura 11 pode se ver o vídeo recebido pelo usuário através da internet por esta câmera.

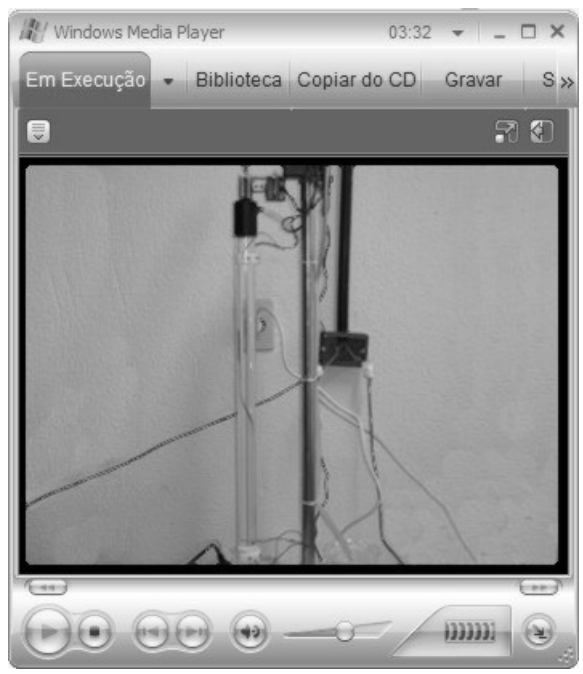

Figura 11: Imagem do experimento vista pela webcam.

A Figura 12 mostra um recorte de um relatório gerado com base nos dados coletados pelo experimento. $\mathrm{O}$ relatório possui todos os cálculos pertinentes à prática laboratorial sobre a determinação da viscosidade de um líquido utilizando o método de Stokes. Além disso, o relatório fornece os cálculos intermediários para determinação da viscosidade do líquido estudado e os cálculos de erros de medição.

A fiducidade ou fidelidade do cálculo é determinada na configuração do experimento e os cálculos de erros são feitos com base na tabela de distribuição de probabilidade estatística conhecida como Student [21]. De acordo a fiducidade escolhida e o número de resultados obtidos. Depois de gerado os resultados o relatório pode ser impresso a qualquer momento mediante autenticação.

\subsubsection{Módulo Administrador}

Este módulo é muito importante e seu uso incorreto pode atrapalhar o bom andamento de todo o ambiente, por isso, o módulo deve tem um controle de segurança mais rígido que o módulo Usuário. Para garantir que seu acesso seja por apenas por usuários do grupo admin autenticados, além de analisar se o usuário ativo e sua autenticação foi realizada com sucesso como é feito no módulo usuário, também se verifica o tipo de usuário que está tentando se autenticar ao sistema. Se os usuários não forem dos tipos citados anteriormente seus acessos ao módulo não são liberados.
Com uso deste sistema é possível realizar todas as operações (inclusão, exclusão, alteração, pesquisa, etc.) para gerenciar os dados referentes aos tipos de esfera, as esferas, aos líquidos que serão o objeto de estudo, as configurações ou parâmetros do experimento a ser utilizado, aos condutores que são os usuários do sistema e às configurações do ambiente.

A janela TipoEsfera controla os tipos de esferas que podem ser utilizadas no experimento. Na configuração do experimento há informação apenas do tipo de esfera que se irá manipular e não sobre cada esfera específica. Isso porque cada esfera tem suas características particulares (massa e diâmetro) mesmo que participem do mesmo grupo. $\mathrm{O}$ grupo deve distinguir esferas de materiais diferentes, como por exemplo, esferas de ferro ou chumbo e também deve distinguir esferas com diâmetros diferentes com erro especificado. Todo este cuidado é importante porque os erros probabilísticos sobre o coeficiente de viscosidade são calculados de acordo com as particularidades das esferas do conjunto utilizado no experimento.

A janela Esfera controla os dados das esferas a serem utilizadas no experimento. As esferas foram pesadas, medidas, cadastradas e separadas por grupos, de acordo com as suas características. Este processo foi feito inicialmente com 100 esferas, podendo-se cadastrar mais esferas a qualquer momento.

A janela Liquido controla os dados dos líquidos que terão a sua viscosidade medida. Como neste experimento há apenas um tubo, o líquido a ser utilizado é selecionado na janela Configuração, assim como o tipo de esferas a qual será submetido.

A janela Configuracao mantém o registro das configurações a serem utilizadas na manipulação do experimento. É possível ter apenas uma configuração ativa num dado instante. Nesta janela determina-se a fidelidade dos valores a serem calculados, o líquido que será utilizado no experimento e o tipo de esferas que serão soltas na superfície do líquido. A troca da configuração, demanda que manualmente troque-se os elementos (e.g., esferas, líquido) envolvidos na realização do experimento.

A janela Condutor é utilizada para manter os usuários que podem utilizar alguma funcionalidade do sistema que necessite de autenticação. Estes usuários podem ser do tipo admin com permissão total às janelas dos módulos Administrador e Usuário ou podem ser do tipo user que podem ter acesso a apenas ao módulo Usuário da ferramenta. Há uma opção nesta janela para poder habilitar/desabilitar um usuário no sistema. Nenhum usuário, seja ele de qualquer tipo, consegue autenticar-se no sistema se ele estiver bloqueado. Há também nesta janela o número de vezes que o usuário pode realizar o experimento, sendo este valor decrementado a cada utilização. 


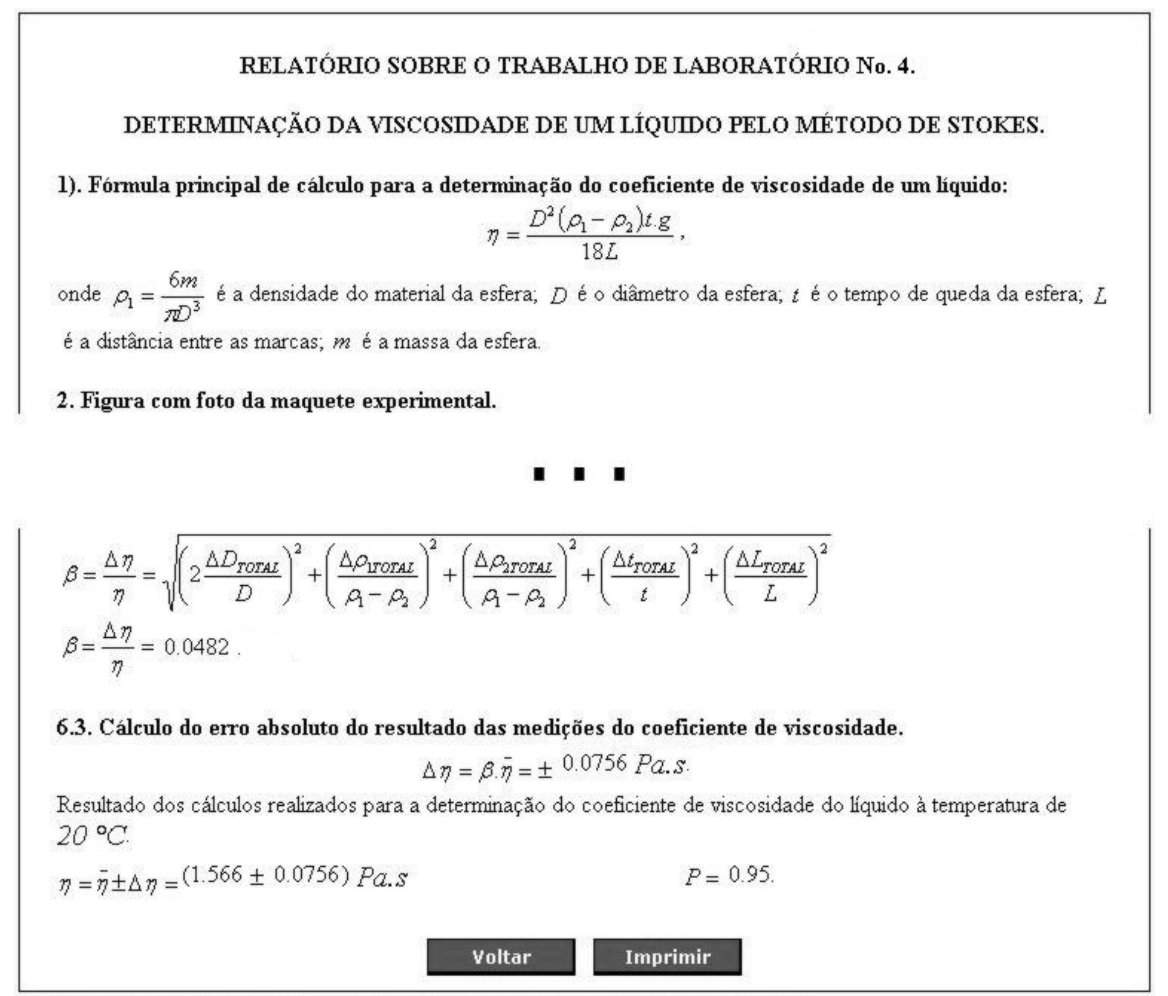

Figura 12 : Recorte do conteúdo do Relatório de Resultados.

Quando o valor chega a zero, o usuário não pode mais utilizar o experimento, mas isso não o impede de acessar o sistema para visualização ou impressão dos resultados já obtidos.

A janela ArquivoIni é utilizada para a alteração dos parâmetros de configuração do arquivo CONFIG.INI. Mesmo que os dados do arquivo tipo Ini seja mais fáceis de serem manipulados do que os dados do banco de dados, a interface facilita ainda mais esta interação, evitando inconsistências com a validação de campos. Com isso, erros como retirada de alguma propriedade, alteração do arquivo errado ou inclusão de valores inválidos não podem acontecer, além de tornar transparente a localização do arquivo para o administrador.

\section{Considerações Finais}

O desenvolvimento do ambiente para a prática remota de aulas laboratoriais de física, especificamente para a determinação da viscosidade de líquidos, constou de 3 etapas: 1) criação da maquete experimental para a determinação de grandezas físicas em estudo; 2) elaboração de um circuito eletrônico para manipular a maquete, de maneira que seja possível obter os resultados necessários ao cálculo dessas grandezas e 3) desenvolvimento de uma ferramenta de software que gerencia o circuito eletrônico, permitindo o acesso e visualização do experimento pela web.

A automatização da prática laboratorial sobre a determinação da viscosidade de líquidos utilizando o método de Stokes será útil, tanto para o Curso de Licenciatura em Física da UESB, curso presencial, como para o Curso de Licenciatura em Física, na modalidade de Educação à Distância, implantado recentemente na instituição. A grande importância da criação deste ambiente para a EaD reside na possibilidade dos alunos do curso de física à distância realizarem as suas práticas laboratoriais de forma análoga ao alunos do curso presencial.

Está em andamento um aprimoramento do ambiente para a EaD apresentado neste artigo, visando uma melhor aproveitamento didático-pedagógico por partes dos alunos. Para tanto, as seguintes modificações serão realizadas:

1) Modelo Modificado da Maquete Experimental Automatizada

Está em construção um modelo modificado da maquete experimental automatizada, apresentada na Figura 2, na qual permitirá que os alunos escolham entre 6 líquidos, e entre tamanhos de esferas, sem a intervenção humana, para o estudo da viscosidade dos líquidos e avaliação dos efeitos dos tubos de diferentes diâmetros 
O novo modelo introduzirá os seguintes componentes de hardware: 1) Conjunto de 6 tubos de vidro transparente de diâmetros diferentes, contendo os líquidos em estudo; 2) 6 motores de passo para controlar os distribuidores individuais de esferas de tamanhos diferentes, que as liberam nos tubos; 3) Motor de passo para o girar de um disco de alumínio, para posicionar cada distribuidor sobre o centro de cada um dos seis tubos da maquete experimental; 4) Conjuntos de sensores de passagem das esferas dentro de cada coluna líquida (diodo emissor de luz/fototransistor) separados por uma distância L; 5) Hardware de controle (drive) para cada motor de passo;

2) Desenvolvimento da Abordagem Computacional para Melhor utilização da Ferramenta para a EaD

Para maior interação entre os alunos será retirado o filtro no qual os estudantes têm acesso apenas a seus experimentos, de forma que estes possam acessar qualquer experimento. Isso demanda alteração da janela "Experimento", para que o aluno só possa alterar ou excluir os seus próprios experimentos.

É necessário acrescentar também na janela "Experimento", e consequentemente no banco de dados, informações para que o aluno possa proceder a escolha do líquido a ser estudado, contido num tubo com dado diâmetro, uma vez que a maquete suportará a troca do mesmo pela internet, assim como a escolha dos tamanhos das esferas que serão utilizadas.

No sistema servidor será adequado o módulo que realiza o controle do motor de passo, a fim de gerenciar 7 motores, sendo 1 para girar o disco de alumínio, posicionar cada um dos distribuidores de esferas de diferentes tamanhos no centro das colunas de líquidos escolhidos, contidos nos tubos com diferentes diâmetros, e os outros 6 para girar os motores acoplados aos respectivos distribuidores de esferas individuais por tamanho.

\section{3) Abordagem Didático-Pedagógica}

a) O modelo modificado da maquete experimental automatizada irá permitir que o aluno, além da determinação da viscosidade de um líquido, explore o método experimental proposto investigando as suas limitações;

b) O giro do disco onde estarão instalados os sistemas distribuidores individuais de esferas de vários tamanhos irá permitir o posicionamento de cada um deles sobre o centro dos tubos de diferentes diâmetros cheios de líquidos translúcidos diferentes. Isto irá permitir ao aluno explorar o modelo teórico, expresso pela equação 5 , investigando a dependência das variáveis e as hipóteses do modelo: o efeito do tipo de esfera frente às distâncias das paredes transversais;

c) Utilizando-se a flexibilidade do modelo modificado da maquete experimental, manipulada remotamente e permitindo-se o acesso de todos os alunos aos resultados de medições realizadas previamente, contidos no banco de dados, poder-se-á realizar um fórum virtual, provido pelo próprio ambiente Web, para o estudo da cinemática e dinâmica de corpos que se movem em meios dissipativos, sob a supervisão dos professores da disciplina.

d) $\mathrm{Na}$ atual abordagem, o ambiente desenvolvido para a EaD gera um relatório com base nos dados coletados pelo experimento que calcula a viscosidade do líquido em estudo pelo método de Stokes, utilizando a equação (4), justa somente quando a esfera cai num meio sem fronteiras (Figura 12). Com a nova abordagem, poderão ser verificadas as limitações do método de Stokes. Através dos dados coletados das medições dos tempos de queda das esferas de massas diferentes, mas de igual densidade, pode-se estabelecer que dependência verifica-se neste ou noutro diapasão de massas.

\section{Referências}

[1] I. N. Oliveira. Projeto de automatização das medições e da elaboração dos dados dos experimentos de física do Laboratório de Física Molecular e Termodinâmica da UESB. Universidade Estadual do Sudoeste da Bahia, 2006.

[2] I. N. Oliveira. Trabalho de Laboratório $\mathrm{N}^{\circ}$ 4: Determinação da viscosidade de um líquido pelo método de Stokes. Universidade Estadual do Sudoeste da Bahia, publicação interna, 2002.

[3] M. H. Nussenzveig. Curso de Física Básica 2. $3^{\text {a }}$ ed. São Paulo, SP: Edgard Blücher, 1933.

[4] M. A. Cavalcante e R. Haag. Corpo negro e determinação experimental da constante de Planck. Revista Brasileira de Ensino de Física. Vol 27(3). ISSN 0102-4744. pp. 343-348. 2005.

[5] E. Schumacher et al. Física Experimental Auxiliada por Laboratório Virtual. XVI Simpósio Nacional do Ensino De Física. 2005.

[6] A. A. Neves. Experimentação Remota para o Ensino de Física. Centro de Referência para o Ensino de Física - UFRGS. Disponível em: $<$ http://www.if.ufrgs.br/cref $>$, acessado em: 04/2009.

[7] S. Paladini. Experimentação Remota como Suporte a Ambientes de Aprendizagem de Física. Centro de Referência para o Ensino de Física. Dissertação de Mestrado do PPG em Engenharia e Gestão do Conhecimento da UFSC. 2008.

[8] M. L. Belloni. Educação a Distância. $3^{\mathrm{a}}$ ed. Campinas, SP: Autores Associados, 2003. 
[9] R. A. F. Castillo. Moodle: Modular Object Oriented Dynamic Learning Environment. Centro de Computação da Universidade Estadual de Campinas. Disponível em: <http://moodle.org $>$, acessado em: 04/2009.

[10] C. Lucena e H. Fuks. Guia AulaNet Home. EduWeb. Laboratório de Engenharia de Software da PUC-Rio. Disponível em: <http://www .eduweb.com.br/downnet/imprensa.htm>, acessado em: 04/2009.

[11] D. F. Oitsuka, T. B. Ferreira e V. H. Rocha. Um Modelo de Suporte à Avaliação Formativa no Ambiente TelEduc. Revista Brasileira de Informática na Educação. Sociedade Brasileira de Computação. 2003.

[12] R. A. Fonseca. e-ProInfo: O ambiente virtual de aprendizagem do MEC. Centro de Computação da Universidade Estadual de Campinas. Disponível em: <http://www.ccuec.unicamp .br/ead/index_html?foco2=Publicacoes/78095/85 2295>, acessado em: 04/2009.

[13] F. G. Capuano, I. V. Idoeta. Elementos de Eletrônica Digital. 29. ed. rev. São Paulo, SP: Érica, 1998.

[14] G. Czajkowski, L. Daynes and M. Wolczko. Automated and Portable Native Code Isolation. Proceedings of the 12th International Symposium on Software Reliability Engineering (ISSRE). 2001.

[15] A. P. Malvino. Eletrônica. 4. ed. Volume 1: Makon Books, 2001.

[16] R. S. Pressman. Engenharia de software. 6. ed. Rio de Janeiro, RJ: Mc Graw-Hill, 2006.

[17] A. Silberschartz, H. F. Korth e S. Sudarshan. Sistema de Banco de Dados. 3 ed. São Paulo, SP: Pearson Makron Books, 2006.

[18] A. Temple e R. F. Mello. JSP, Servlets e J2EE. Versão 1.2. GNU Free Documentation License, 2004.

[19] M. Ghaderi and S. Keshav. Multimedia Messaging Service: System Description and Performance Analysis. Proceedings in First International Conference on Wireless Internet (WICON). pages 198-205, 2005.

[20] H. M. Deitel. Java Como Programar. 6. ed. São Paulo, SP: Pearson Prentice Hall, 2003.

[21] R. A. Fisher. Applications of "Student's" distribution. Metron 5: pages 90-104, 1925. 